\title{
Design and Modeling of Schmitt Trigger-based Sub- Threshold 8T SRAM for Low Power Applications
}

\author{
Mahipal Dargupally \\ M.Tech (VLSI System Design) \\ B V Raju Institute Of \\ Technology, Dept.of ECE, \\ (CVD), Narsapur, Medak Dt, \\ Telangana, India
}

\author{
T. Vasudeva Reddy \\ Assoc. Professor, B V Raju \\ Institute Of Technology, \\ Dept. of ECE, (CVD), Narsapur, \\ Medak Dt, Telangana, India
}

\author{
Udary Gnaneshwara \\ Chary \\ Assist. Professor, B V Raju \\ Institute Of Technology, \\ Dept.of ECE, (CVD), Narsapur, \\ Medak Dt, Telangana, India
}

\begin{abstract}
This paper presents the design of Schmitt trigger-based 8T SRAM Architecture for low power sub-threshold (or) nearthreshold CMOS SRAM for power constrained Applications. Power Consumption, Power Dissipation and Leakage Power are the main factors in the IC Design. Memory unit is the primary block in design of any chip like Micro Processor and Micro Controller. As SRAMs comprise a significant percentage of the area and power for many digital chips and leakage can dominate total chip leakage. The proposed paper used to reduce the leakage power by using High- $\mathrm{V}_{\text {th }} \mathrm{nMOS}$ as pull-down transistors for standard 6T SRAM. This paper demonstrates the Architecture Design and Analysis of 256bitcell $8 \mathrm{~T}$ SRAM in $45 \mathrm{~nm}$ technology. The design implementation and analysis is performed using $45 \mathrm{~nm}$ CMOS technology in CADENCE IDE.
\end{abstract}

\section{Keywords}

8T SRAM, Sub-Threshold, Low power, Low Leakage

\section{INTRODUCTION}

The Digital sub-threshold circuit design has become a very promising method for ultra-low power applications. Circuits operating in the sub-threshold region utilize a supply voltage $\left(\mathrm{V}_{\mathrm{DD}}\right)$ that is close to or even less than the threshold voltages $\left(\mathrm{V}_{\mathrm{th}}\right)$ of the transistors. This low $\mathrm{V}_{\mathrm{DD}}$ operation results in ultra low-power dissipation. The circuit operating from strong inversion, moderate inversion regions to weak inversion region can be known as sub-threshold operating region[4].

In Sub-threshold region standard 6T-SRAM shows weak read and write stabilities[5]. the performance depend on static noise margin (SNM). Static Random Access Memory(SRAM) remain to be one of the most paramount and integral memory technologies today mainly because of their robustness and ease at manufacturing. SRAM memory cell better than DRAM as it can perform at low power and can store number of bits .

The rising demand of portable electronics for computing, communication and other applications has necessitated an improved battery life and low power consumption. Subthreshold SRAM provides an advantage in minimizing total memory energy consumption and providing compatibility with minimum-energy sub-threshold logic[6].

\section{SRAM ARCHITECTURE 2.1 Proposed 8T SRAM}

The Static random access memory (SRAM) is a type of volatile semiconductor memory to store binary logic ' 1 ' and ' 0 ' bits. SRAM uses bistable latching circuitry made of
Transistors/MOSFETS to store each bit. An SRAM is designed to fill two needs: to provide a direct interface with the CPU at speeds not attainable by DRAMs and to replace DRAMs in systems that require very low power consumption. The design requirement is such that pMOS OFF- state current should be more than the pull-down nMOS transistor leakage current for maintaining data " 1 " reliably. With increasing process variations and exponential dependence of the subthreshold current on the threshold voltage, satisfying this design requirement across different process, voltage, and temperature (PVT) conditions may be challenging.

The proposed Architecture is designed using Schmitt triggerbased 8T SRAM bitcell illustrated in Fig.[1]. It is similar to the standard 6T SRAM cell, however it is used High- $\mathrm{V}_{\text {th }}$ nMOS in this design[4]. Two sleep transistors are used in pull down path to minimize the leakage power[2]. These Transistors used for self correcting feedback to achieve stable operation. This cell also reduces dynamic power in active mode.

\section{A. WRITE OPERATION}

In the write operation, WL is enabled to activate the access transistors for data transfer. The proper write operation can be done successfully by consideration of Aspect ratio(W/L) of nMOS and pMOS[6]. In sizing of transistors in SRAM bit cell nMOS should win the ratio fight with pMOS. If we want to write data' 1 ' making the $\mathrm{WL}=1$ and one of the bitline keeping High $\mathrm{BL}$ (or) $\mathrm{BLB}=1$ and other is 0. Then the value is been written as ' 1 ' at node 'Q' and its complement at node 'QB'.

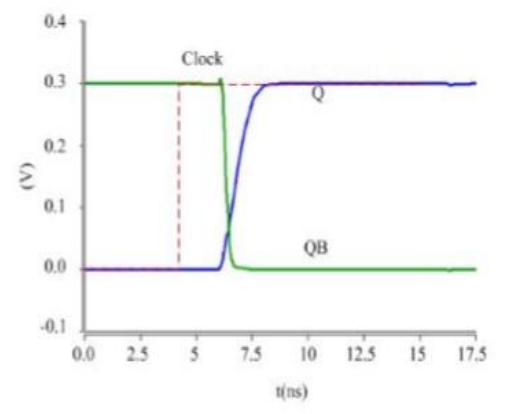

Fig.1 Schmitt Trigger-based 8T SRAM

\section{B. READ OPERATION}

In the read operation, BL and BLB are precharged and $\mathrm{WL}$ is enabled then one of the Bitline(BL/BLB) is making low . The read value can be observed by enabling the Sense Amplifier. 


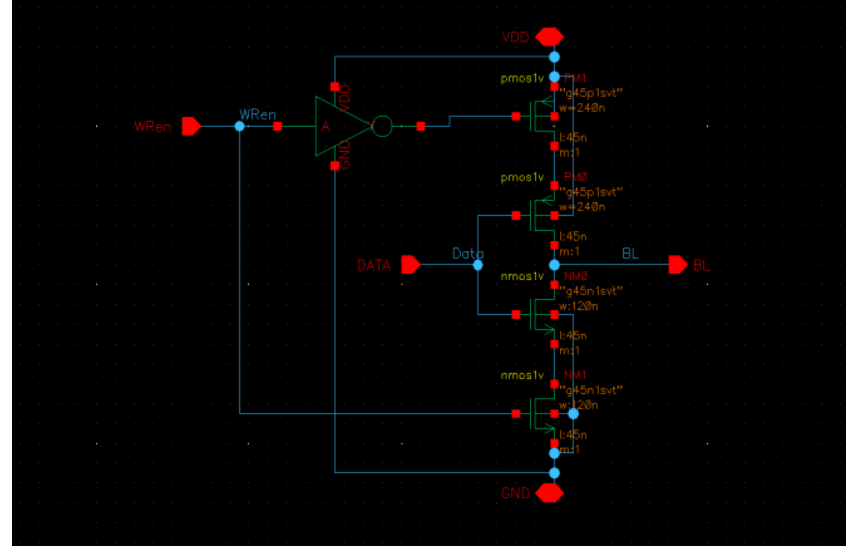

Fig.2 The simulation result of a single cell write operation

The written data can be stored at node ' $Q$ ' and its complement at 'QB' in standby mode. this data can be with held at the node till the read operation being accessed. here the leakage has been minimized by using the sleep transistors in pull down path and effectively read by acting as feedback transistors.

\subsection{Sense Amplifier}

The voltage mode sense amplifier is used for designing. This is the Enhanced Positive Feedback sense amplifier is using to operate the read operation effectively and it can reduces the bit line capacitances by decoupling the nMOS transistors in design shown in Fig.3. The written data can be enhanced effectively here[8]. This design operated at $0.4 \mathrm{~V}$ and power dissipation is $942.2 \mathrm{pW}$.
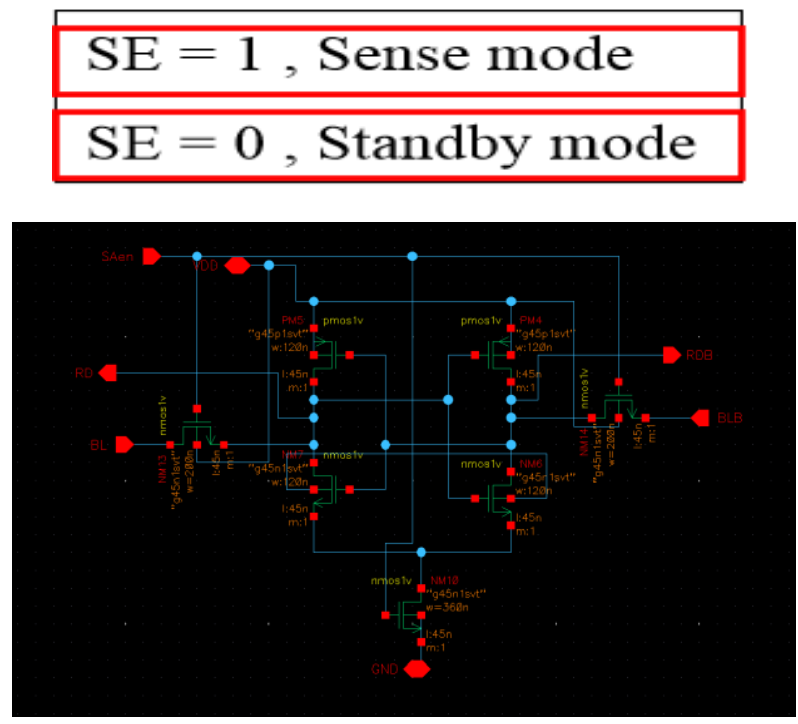

Fig.3 Enhanced Positive Feedback Sense Amplifier

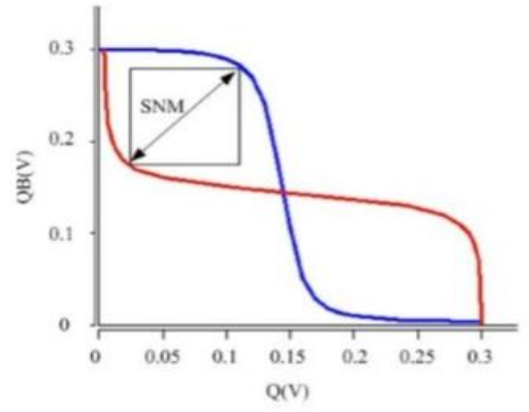

Fig.3 SNM of the proposed SRAM

\subsection{Data Write Circuit}

Write operation into the memory cell can be done only when the write enable ON. This enable operation can be easily understood by the following circuit Fig.3. Write operation into the memory cell can be done only when the write enable ON This enable operation can be easily understood by the following circuit. The design being operate at $0.4 \mathrm{~V}$ and observed delay and power $4.79 \mathrm{nS}, 1.38 \mathrm{nW}$ respectively.

\subsection{Decoder and Multiplexer}

The proposed Architecture is also consist of decoder and multiplexers for Memory Array operation designed. The 4to16 Decoder is designed using 4input nor-gate design and multiplexer16x 1 is using four $4 \times 1$ mux with nand-gate based design for minimizing area and power.

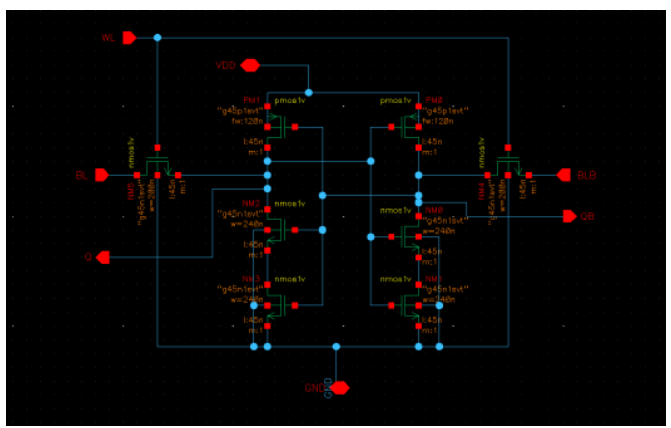

Fig.4 Data Write Circuit

\section{ARCITECTURE OF 256BIT SCHMITT TRIGGER-BASED 8T SRAM}

The proposed architecture of 256bit ST-based 8T SRAM cells is designed using address decoder, pre-charge circuitry, data write circuitry, sense amplifiers and 16x16 SRAM array in $45 \mathrm{~nm}$ technology. The Fig.5 shows the design implementation symbol for proposed architecture. The Fig.8 illustrates the implementation of single bit ST-based 8T cell. The block diagram of SRAM memory with all input signals, pre-charge, write enable, sense amplifier enable, 16x16 memory array has illustrated in Fig.8. 


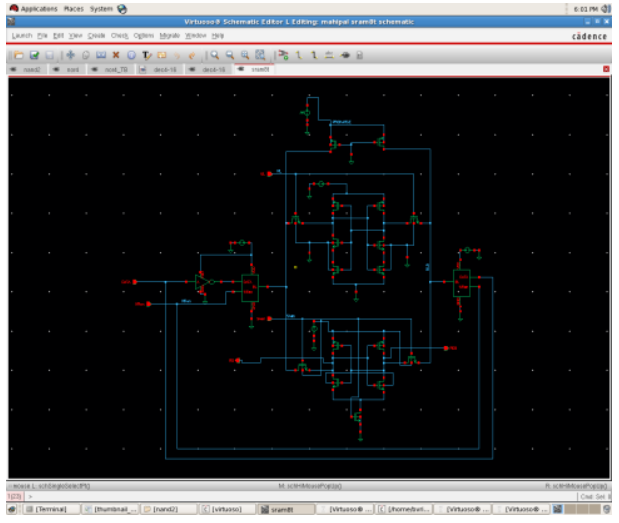

Fig.7 single bit schmitt trigger-based 8T SRAM

\section{SIMMULATION RESULT}

The Fig.9 illustrating the operation result of proposed Architecture. This paper explores the design of Schmitt trigger-based 8T SRAM bit cell. observation of operations and analysis of different parameters like power consumption(PC), leakage power(LP) and delay in the sub threshold region. The design has manipulated for different voltage supplies measurements have been observed over the range of voltages $0.8 \mathrm{~V}$ down to the sub threshold region in $45 \mathrm{~nm}$ technology library. The CADENCE VIRTUOSO SCHEMATIC EDITOR was used for designing and analyzing[10]. The Table.1 shows the operated result.

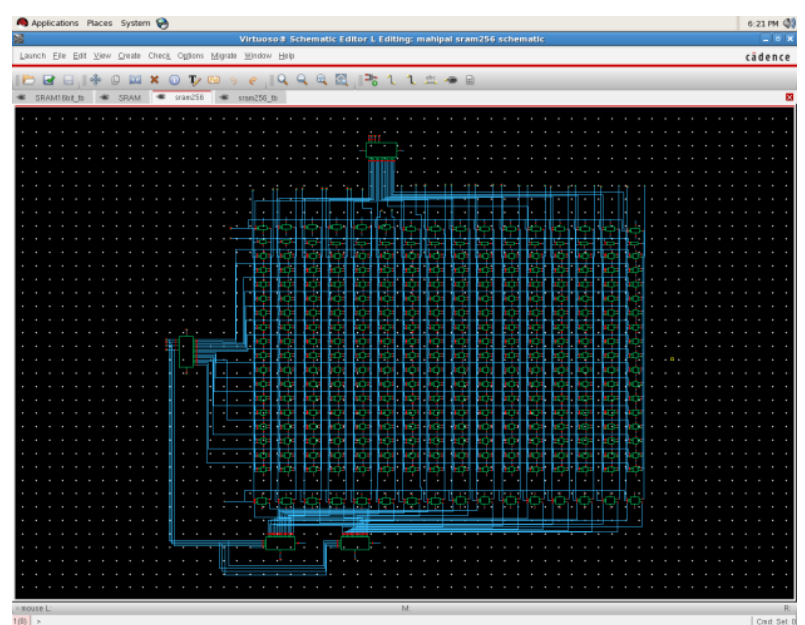

Fig.8 256Bit memory array

Table.1: operation result of SRAM Cell

\begin{tabular}{|c|c|c|c|}
\hline $\mathbf{V}_{\text {DD }}$ & Single bit & 16bit & 256bit \\
\hline $\begin{array}{c}\text { Power } \\
\text { Consumption }\end{array}$ & $12.765 \mathrm{nW}$ & $317.9 \mathrm{nW}$ & $474.6 \mathrm{nW}$ \\
\hline Leakage Power & $0.497 \mathrm{nW}$ & $4.74 \mathrm{nW}$ & $7.98 \mathrm{nW}$ \\
\hline DELAY & $114.6 \mathrm{~ns}$ & $156.4 \mathrm{~ns}$ & $168.9 \mathrm{~ns}$ \\
\hline
\end{tabular}

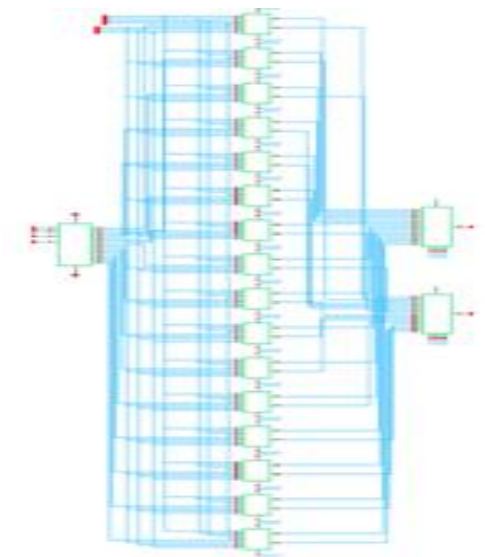

Fig.5 256Bit Cell Architecture Symbol

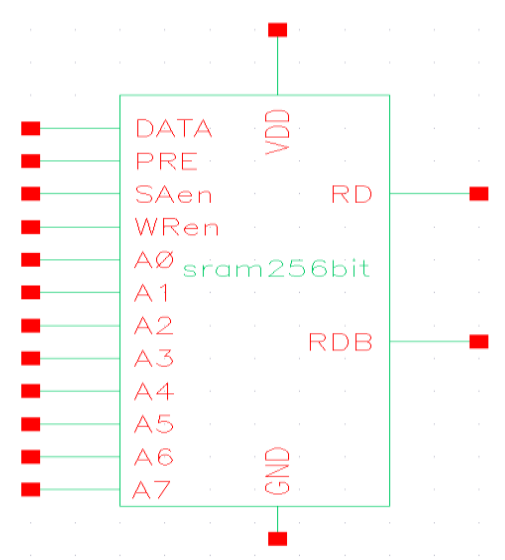

Fig.6 Proposed Architecture

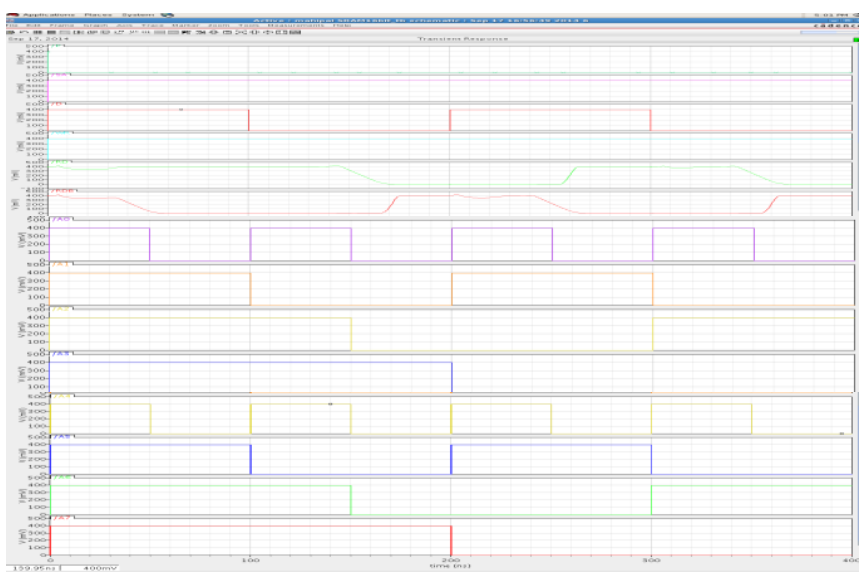

Fig.9 Performance characteristics of operation

\section{CONCLUSION}

This study implemented a 256b Schmitt Trigger-Based SubThreshold 8T SRAM in a $45 \mathrm{~nm}$ CMOS technology with a High-Vth nMOS and sleep transistors in pull down path. It has been reduced a leakage power in high rate. architecture design complexity reduced with appropriate Module selection for sixteen ST-based 8T bit cells in bit-interleaving scheme. We simulated the single bit SRAM with a normal Vth NMOS in the Write path observed the power consumption of $1.15 \mathrm{nW}$ and in High-Vth nMOS the power consumption reduced to $117.7 \mathrm{pW}$. The simulation results of entire Architecture power 
consumption is $474 \mathrm{nW}$ with an operation frequency of $6 \mathrm{MHz}$ at $400 \mathrm{mV}$ was achieved. The standby power dissipation is $7.98 \mathrm{nW}$. Hence the design has achieved low power operation without degrading the performance.

\section{ACKNOWLEDGEMENT}

The authors would like to thank Dr.I.A.Pasha,HOD, ECE Department, BVRIT. This work was done in CVD(CENTER FOR VLSI DESIGN) Lab. They express gratitude to Mr.I.B.K Raju, Assoc.prof and They also express thanks to the management of BVRIT, Narsapur, medak and parents, friends \& colleagues.

\section{REFERENCES}

[1] Inventors: Ching-Te Chuang, Chien-Yu Hsieh, MingLong Fan, Pi-Ho Hu, Pin Su "Schmitt Trigger-based FINFET SRAM cell" Patent Publication number US20120014171.

[2] Rajani H.P. and Srimannarayan Kulkarni "NOVEL SLEEP TRANSISTOR TECHNIQUES FOR LOW LEAKAGE POWER PERIPHERAL CIRCUITS" International Journal of VLSI design \& Communication Systems (VLSICS) Vol.3, No.4, August 2012.

[3] Mahipal Dargupally, T.Vasudeva Reddy "Sub-threshold SRAM bit cell topologies for ultra low power applications" International Journal of Scientific \& Engineering Research, Volume 5, Issue 8,August-2014 ISSN 2229-5518.

[4] Wei-Bin Yang, Chi-Hsiung Wang, I-Ting Chuo, HuangHsuan Hsu" A 300 mV 10 MHz 4 kb 10T Sub-threshold SRAM for Ultralow-Power Application" 2012 IEEE International Symposium on Intelligent Signal Processing and Communication Systems (ISPACS 2012) November 4-7, 2012.

[5] David J. Comer, Senior Member, IEEE, and Donald T. Comer "Operation of Analog MOS Circuits in the Weak or Moderate Inversion Region" IEEE TRANSACTIONS ON EDUCATION, VOL. 47, NO. 4, NOVEMBER 2004.
[6] Alice Wang Benton H. Calhoun Anantha P. Chandrakasan "Sub-threshold Design for Ultra LowPower Systems "SERIES ON INTEGRATED CIRCUITS AND SYSTEMS

[7] Zhiyu Liu and Volkan Kursun "Characterization of a Novel Nine-Transistor SRAM Cell" IEEE TRANSACTIONS ON VERY LARGE SCALE INTEGRATION (VLSI) SYSTEMS, VOL. 16, NO. 4, APRIL 2008

[8] "Sense Amplifier for SRAM" by Professor: Der-Chen Huang

[9] Neil H. E. Weste David Harries Ayan Banerjee "CMOS VLSI Design" Third edition.

[10] Cadence® Virtuoso® Spectre ${ }^{\circledR}$ Circuit Simulator, 2009.

\section{AUTHOR'S PROFILE}

Mahipal Dargupally is research student, pursuing M.Tech in VLSI System Design from B.V.Raju Intitute of Technology, completed B.Tech from JNTUH, Hyderabad. His Area of interest is Low power VLSI Design, Memory design and Logic circuits.

T.Vasudeva Reddy was born in 2nd FEB 1979. He received his B.TECH from Madras University, Tamilnadu In 2002. And M.TECH from JNTU Hyderabad, AP, in 2008. He is an Associate Professor, Dept.of Electronics \& Communication Engineering in B.V.Raju Institute Of Technology, Narsapur ,Medak Dt,. Presently, he is pursuing $\mathrm{PhD}$ on the topic of low power memory cell design. He had 12 years of teaching experience. His research interests are Low power VLSI design.

Udary Gnaneshwara Chary obtained $M$. Tech degree in VLSI System Design from VNR Vignan Jyothi Institute of Technology. Presently, he is pursuing PhD from JNTU Hyderabad. $\mathrm{He}$ is an Assistant Professor in B.V.Raju Institute of technology Narsapur, Medak. He had 8years of teaching experience. His research interests include CMOS Analog design. 